Article

\title{
Social-Aware Peer Selection for Device-to-Device Communications in Dense Small-Cell Networks
}

\author{
Aamir Nadeem and Ho-Shin Cho * \\ School of Electronics Engineering, Kyungpook National University, Daegu 41566, Korea; nadeem@knu.ac.kr \\ * Correspondence: hscho@ee.knu.ac.kr
}

Received: 14 May 2019; Accepted: 10 June 2019; Published: 13 June 2019

\begin{abstract}
Device-to-device communications refer to the emerging paradigm that permits direct communication between cellular users that are in close physical and social proximity. It is expected to become an essential aspect of the future $5 \mathrm{G}$ cellular communications system. As a prerequisite for device-to-device communications, a user has to select another user in its proximity that has the desired information/service and is willing to share it. In this paper, we propose a method for peer selection in dense small-cell device-to-device networks that uses multi-attribute decision modeling. The method exploits both the physical and social characteristics of the user equipment to find the most suitable peer for device-to-device communications. We assume hexagonal small-cell and macro-cell architectures with a small-cell/macro-cell base station with multiple user equipments in its coverage area to evaluate the proposed scheme's performance. The small-/macro-cell base station exploits various social and physical attributes to rank peers and selects the best one for device-to-device communication with the requesting user. The numerical results demonstrate the proposed algorithm's efficiency in terms of computational time, selection of the best peer, throughput, and energy efficiency of device-to-device communications.
\end{abstract}

Keywords: device-to-device communications; user equipment; peer selection; multi-attribute decision modeling

\section{Introduction and Contribution}

Over the last decade, there has been tremendous growth in the demand for data and other network applications and services, largely arising from mobile devices. This has led to a scarcity of spectrum and capacity in cellular systems. Despite the revolution in radio access and networking technologies, the current cellular system is becoming heavily loaded [1] — if this trend continues, the scarcity will worsen-it is projected to increase seven-fold in 2016-2021 [2]. This has motivated researchers to find new mechanisms to offload traffic from cellular base stations (BS) and fulfill ever-increasing customer demands.

Network densification through small-cell networks (SCNs) is a promising solution to the aforementioned issue. The main concept of SCN is to overlay several small-cell base stations (SCBSs) within an existing legacy cellular system. This can efficiently reuse the spectrum and improve both coverage and capacity, particularly in densely populated urban areas. However, the SCN model has certain challenges, such as resource allocation, interference mitigation, and backhaul network management that need to be addressed separately [3,4]. Similarly, device-to-device (D2D) communications is another exciting approach that has received substantial research attention. This communication system can offload traffic from legacy macro-cell base stations (MCBS) by allowing proximate user equipments (UEs) to communicate directly [5]. Moreover, it can improve the coverage, capacity, and spectrum utilization of a cellular system by efficiently reusing resources and mitigating spectrum congestion. Both D2D and SCN are expected to be integral parts of future $5 \mathrm{G}$ cellular communication systems $[4,6]$. As these two 
paradigms aim to provide short-range communications with low-cost and power, they can be merged to provide more efficient D2D communications. The combined model can significantly facilitate D2D peer selection, link establishment, and communication.

Before establishing a D2D link, D2D user equipment (DUE) has to discover and select a suitable peer that both has the required services, and is willing to serve the requesting UE. This process can be performed either with or without the assistance of the MCBS. The former method can improve the peer discovery process at the cost of higher signaling overhead. Conversely, the latter approach can achieve scalability and out-of-coverage discovery but lacks synchronization and collision avoidance [5]. SCN-based D2D communications can acquire the merits of both designs and overcome some of their individual challenges [4].

D2D communications rely on the cooperation between the UEs where a target UE will serve the requesting UE with its desired data or application. This involves the use of resources of the serving UE, which may not be possible due to users' selfish nature. Consequently, users may not always be willing to provide resources to unknown fellow users. To overcome this issue, many recent works have combined users' social parameters with their physical parameters. This has significantly improved users' willingness to serve people in their social circle. However, using users' social information leads to privacy concerns, particularly in distributed D2D communications, which has not been adequately addressed in most of the literature.

The purpose of this work is to explore the impact of social and physical attributes on D2D peer selection in a novel manner. The ultimate goal is to improve the peer selection process without compromising on user privacy as in distributed selection methods, and without adding any burden on legacy MCBS, as in centralized methods. Moreover, various social and physical attributes have different impacts on D2D peer selection. Therefore, we need a method that can integrate these diversified attributes and compute a joint peer selection metric. Motivated by these objectives, our proposed work has the following main contributions:

1. Presents a hybrid architecture that combines two emerging technologies, i.e., D2D and SCN, to facilitate the D2D peer selection process. Unlike traditional methods that either consider direct or legacy MCBS-assisted device discovery, the proposed method assumes assistance from the SCBS.

2. Proposes a social-aware peer selection algorithm for D2D communications using a modified version of a well-known multi-attribute decision modeling (MADM) algorithm known as the technique for order of preference by similarity to ideal solution (TOPSIS) [7]. This algorithm efficiently exploits UEs' physical and social attributes and determines their cumulative closeness coefficients based on which it selects a D2D peer. When there is no friendly user in proximity, the proposed method performs peer selection based on the similarity in the users' interest.

3. Utilizes the cumulative closeness coefficient to compute the signal-to-noise ratio, data rate, and finally the throughput of D2D communications.

4. Shows the performance of the proposed scheme in terms of the algorithm's computational time, best peer selection, throughput, and the energy efficiency of the D2D communications.

The rest of the paper is divided as follows: Section 2 reviews the relevant literature and highlights some issues; Section 3 discusses the system model and parameter definitions; Section 4 is related to the formulation of the social-aware D2D peer selection algorithm; Section 5 discusses the simulation setup and performance evaluation; and the final section summarizes the discussion with a conclusion and presents directions for future work.

\section{Related Works}

The recent literature consists of various methods for D2D peer selection based on different parameters. Zufan et al. [1] proposed a cooperative model for D2D communications that jointly considered the social and physical relationship between users and developed a stable matching-theory based algorithm for D2D peer selection. However, this work considered a traditional macro-cell 
scenario in which the peer selection process increased the burden on the MCBS in terms of signaling and social information management. Wang et al. proposed a user-pairing scheme based on resource sharing between cellular and D2D users [8]. This method aimed to improve resource utilization by selecting a suitable D2D partner for each cellular user equipment (CUE) using a bipartite graph. Similarly, Liu et al. [9] utilized a Voronoi diagram to propose a scheme for UE pairing in D2D communications. Users located in an adjacent area in the diagram were allowed to establish a D2D connection. Power consumption and interference were reduced as the scheme permitted short-range D2D communications between neighboring UEs. However, neither of the methods in [8] and [9] integrated the SCN paradigm. Similarly, the social aspects of users were not considered, which led to the unwillingness of a user to perform D2D communications with unknown fellow users.

Mao et al. proposed a joint social-position relationship based cooperative scheme that exploited both social and location parameters [10]. A partner-selection algorithm with low complexity was derived using a joint closeness metric, which determined a two-layer (i.e., social position) relationship between UEs in a unified manner. The closeness metric values were calculated for the available UEs and sorted to select the most suitable peer for D2D communications. However, the proposed peer selection method was distributed, which increased the energy consumption of UEs. Moreover, the social and physical parameters were manipulated by UEs, which led to privacy concerns. Prasad et al. proposed an application-aware peer discovery algorithm that exploited cloud-based social proximity [11]. The algorithm was based on cloud-assistance for computational offloading, which ensured that the method was energy efficient. Moreover, the proposed method significantly improved the peer discovery process compared to traditional peer discovery schemes. However, the algorithm was not suitable for generic D2D scenarios as it assumed that users had similar interests and the same application subscriptions from the cloud.

Y. Gao et al. recently proposed a dynamic peer selection method with novel social-aware spectrumpower trading for D2D communications [12]. The authors aimed to maximize the energy efficiency by considering the quality of service (QoS) and the minimum rate constraints. They developed two communication modes for cooperative D2D communications between cellular edge users and D2D transmitters, i.e., the D2D link mode and idle mode. A framework was formulated based on their proposed D2D modes to optimize the peer and mode selection in cooperative D2D communications. The authors asserted the notion that as the amount of D2D transmit power was determined by the social relationship between DUEs, a D2D pair can likewise gain a certain level of spectrum resource from cellular UEs based on the strength of their social relationship. The results demonstrated the efficiency of their proposed scheme in terms of energy efficiency and privacy protection. However, their proposed method had higher complexity. Moreover, D2D peer selection was centralized, which increased the load on legacy-based stations.

\section{System Description}

In this section, we describe the system model along with the social and physical proximity layers. Moreover, we define various social and physical parameters used in the proposed algorithm.

\subsection{System Model}

In this paper, we mainly focus on the performance evaluation of our proposed algorithm in small-cell scenarios. However, for comparison with the macro-cell in terms of energy efficiency, we also perform simulations of macro-cell scenarios by changing the coverage area and transmission power. We assume a set of UEs that consists of DUEs and CUEs. Both DUE and CUE have the capability to communicate through the base station. However, we assume DUE as the one that has the desired data/service needed by the requesting UE. Hence, it could be selected as the D2D peer. On the other hand, CUE cannot be selected as the D2D peer because it does not have the desired service/data. The CUEs are allocated with orthogonal sub-channels while the DUEs reuse the same sub-channels for D2D communications. Hence, the CUEs do not interfere with each other. Each D2D pair reuses 
the spectrum resource of a CUE. The BS (SCBS/MCBS) ensures that the resource of a CUE is reused by exactly one D2D pair to mitigate the interference among DUEs. However, interference can occur between CUEs and DUEs because they share the same spectrum resource. In addition, a Rayleigh fading channel model is assumed for the proposed system [13].

We assume that the BS (SCBS/MCBS) can get the social attributes of UEs while it knows their physical locations. When it receives a request for D2D communications from a UE, it constructs a separate decision matrix for the social attributes of the UEs denoted by $\mathrm{M}_{D S}$, and physical attributes denoted by $\mathrm{M}_{D P}$, as presented generically in (5). Before applying the TOPSIS algorithm, the UEs with no social friendships are excluded from the $\mathrm{M}_{D S}$. Similarly, UEs whose distance from the requesting UE is greater than the minimum D2D distance threshold (i.e., $50 \mathrm{~m}$ ) are omitted from the $\mathrm{M}_{D P}$. These significantly simplify the peer selection task. A modified version of the TOPSIS algorithm is then applied to both the $\mathrm{M}_{D S}$ and $\mathrm{M}_{D P}$ to compute the closeness coefficient for social and physical attributes denoted by $\delta_{s}$ and $\delta_{p}$, respectively. The two closeness coefficients are added together to get the cumulative closeness coefficient denoted by $\Delta$, which is then used to calculate the signal-to-noise ratio and the data rate for the D2D communications as given in (15) and (16), respectively. Finally, we use the calculated data rate to compute the throughput and energy efficiency for D2D communications as given in (17) and (18) respectively. The BS probes the candidate's UEs for D2D communications. It shares the information of those who are ready for D2D communications with the requesting UE. Moreover, it also shares the information of the requesting UE with its D2D peer, and hence, they establish a direct link for D2D communications. If there is no UE with social friendship in the $\mathrm{M}_{D S}$, the proposed method selects D2D peers based on the similarity in their interests.

As social-aware D2D communications exploit the attributes of users in both social and physical domains. Therefore, we divided our system model into two layers-a social proximity layer (SPL) and a physical proximity layer (PPL) - (as shown in Figure 1) to model their social relationships and physical constraints. The next subsection briefly discusses both the layers of our model and the way the model reflects the corresponding attributes.

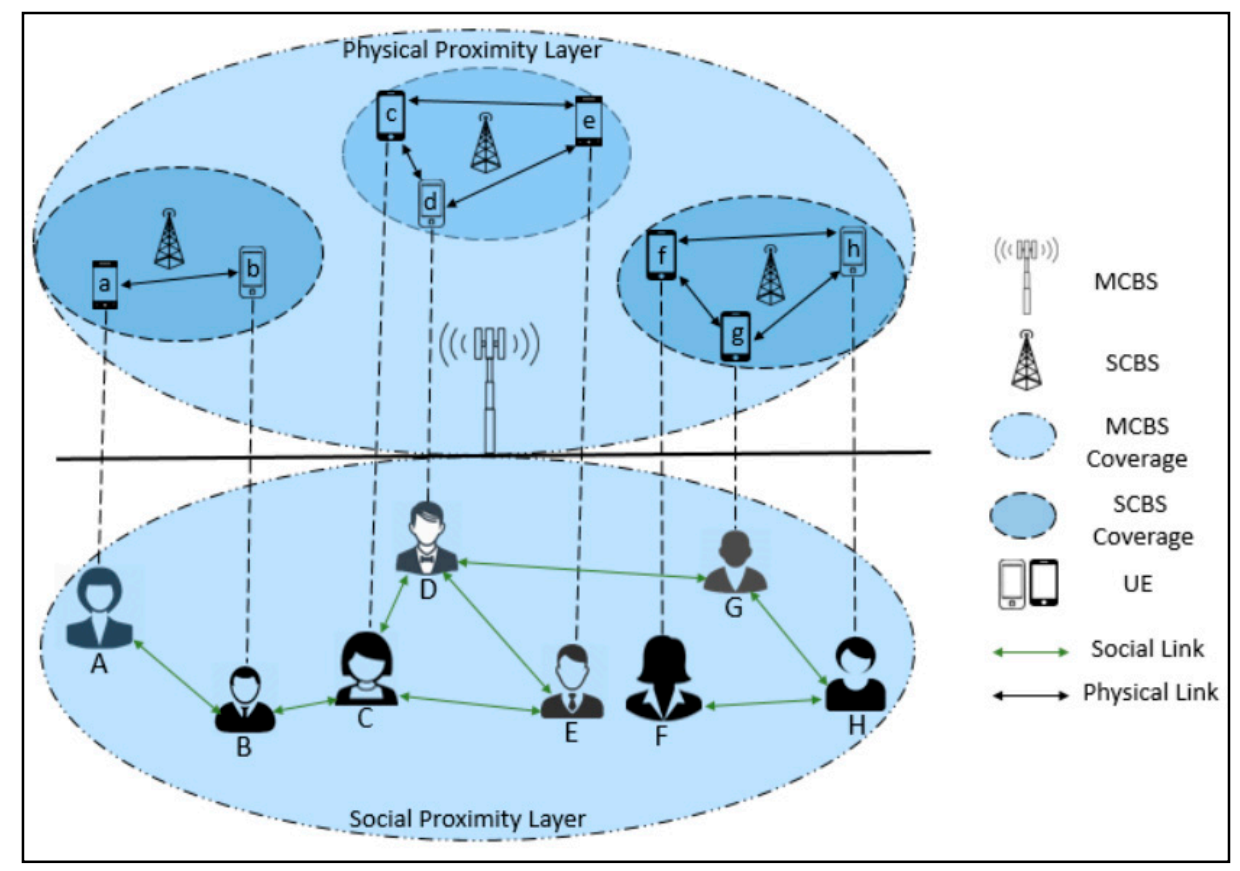

Figure 1. The D2D architecture with social and physical proximity layers.

\subsubsection{Social Proximity Layer}

The SPL characterizes the social attributes of users existing in the social networks. These attributes determine the possibility of pairing between two users for D2D communications. SPL can be modeled 
using the weighted directional graph GSPL = VSPL $\varepsilon S P L$ [14], where VSPL is the vertex set that represents the users (from $\mathrm{A}$ to $\mathrm{H}$ ) and $\varepsilon S P L$ is the edge set that represents the social link between them as shown (with green double arrows) in Figure 1. Human users have different levels of social intimacy with each other, which affect their interaction and communication behavior. Therefore, the social proximity between them is incorporated in the D2D peer selection, which improves the user's willingness to cooperate with others and enhances the overall D2D performance. In the subsequent sections, we represent the transmitter DUE with $T x$ and the receiver DUE with $R x$.

\subsubsection{Physical Proximity Layer}

The PPL describes the physical and network constraints of DUEs. It can also be modeled using the directional weighted graph GPPL = VPPL $\varepsilon$ PPL [14], where the vertices set, VPPL, denotes the DUEs (from a to h) while the edges set, $\varepsilon$ PPL, denotes the physical link between the DUEs as shown (with black double arrows) in Figure 1. The existence of the physical edge $\varepsilon$ PPL between DUEs validates the feasibility of a D2D link between them. As we assume that the SCBS knows the locations of the users, it can determine if the two users are in physical proximity to each other or not. There are four possible D2D scenarios based on the locations of $T x$ and $R x$ :

a. Tx and $R x$ are connected to the same SCBS.

b. Tx and $R x$ are connected to different SCBSs.

c. Tx is connected to the SCBS and Rx to the MCBS.

d. Tx and $R x$ are connected to MCBS.

As D2D refers to the communication between the UEs located in proximity, for simplicity, we assume that the Tx and $R x$ DUEs are connected to the same SCBS.

\subsection{Parameters' Definition}

In the following subsections, we define various parameters for the proposed two-layer system model. The list of notations and acronyms for various parameters and variables used in the paper is given in Table 1 at the end of this section.

Table 1. List of notations and acronyms.

\begin{tabular}{ll}
\hline Symbol/Acronym & Description \\
\hline$Q$ & Number of attributes (social/physical) \\
\hline$\alpha_{Q}^{s / p}$ & Q $_{\text {th }}$ social/physical attribute \\
\hline$A_{(i, j)}$ & Value of attribute $\alpha_{j}$ for $\mathrm{UE}_{i}$ \\
\hline$\| A_{w(i, j)}^{\text {des } / \text { undes } \|}$ & Normalized weighted desired/undesired attribute value \\
\hline $\mathbf{M}_{D}$ & Decision matrix \\
\hline $\mathbf{M}_{D S / D P}$ & Decision matrix for social/physical attributes \\
\hline$T x / R x$ & Transmitter/Receiver DUE \\
\hline$D_{(T x, R x)}$ & Distance between $T x$ and $R x$ \\
\hline$d_{t h}$ & Distance threshold \\
\hline$m$ & Number of UEs \\
\hline$W$ & Weight of the attribute \\
\hline$I_{j}^{+} / I_{j}^{-}$ & Ideal positive and negative solution for $\alpha_{j}$ \\
\hline$D I_{j}^{+} / D I_{j}^{-}$ & Distance of $\alpha_{j}$ from positive/negative ideal solutions \\
\hline$\delta_{i(s, p)}$ & Closeness coefficient for social/physical attributes of UE \\
\hline$H_{(T x, R x)}$ & Channel model between $T x$ and $R x$ \\
\hline
\end{tabular}


Table 1. Cont.

\begin{tabular}{|c|c|}
\hline Symbol/Acronym & Description \\
\hline$h_{(T x, R X)}$ & Rayleigh coefficient \\
\hline$\beta$ & Path loss exponent \\
\hline$\Omega_{U E}$ & Set of UEs \\
\hline $\mathrm{G}_{S P L / P P L}$ & Weighted directional graph for social/physical proximity layer \\
\hline $\mathrm{V}_{S P L / P P L}$ & Vertex set for social/physical proximity layer \\
\hline$\varepsilon_{S P L / P P L}$ & Edge set for social/physical proximity layer \\
\hline SFI & Social friendship index \\
\hline SCI & Social closeness index \\
\hline$\Phi_{T x / R X}$ & Friend list of $T x / R x$ \\
\hline ISI & Interest similarity index \\
\hline$\Psi_{T x / R x}$ & Interest list of $T x / R x$ (individual) \\
\hline$\Psi_{T x R x}$ & Mutual interest list of $T x$ and $R x$ \\
\hline$\Upsilon_{(T x, R x)}$ & Encounter duration between $T x$ and $R x$ \\
\hline$e$ & Number of encounters \\
\hline$t_{i(T x, R x)}$ & Time of $i$ th encounter between $T x$ and $R x$ \\
\hline$\Gamma$ & Total sample time \\
\hline$X_{T x / R x}, \theta_{T x / R x}$ & Polar coordinates of $T x$ and $R x$ \\
\hline$\gamma$ & Signal-to-noise ratio \\
\hline$P_{(T x)}$ & Transmission power of $T x$ \\
\hline$\Delta_{(T x, R x)}$ & Cumulative closeness coefficient between $T x$ and $R x$ \\
\hline$\sigma$ & Variance \\
\hline$R_{(T x, R x)}$ & Data rate for D2D communications between $T x$ and $R x$ \\
\hline$B W$ & Available bandwidth \\
\hline $\mathfrak{J}_{(T x, R x)}$ & Throughput of D2D communication between $T x$ and $R x$ \\
\hline $\mathrm{T}_{T x}$ & Tx transmission time \\
\hline$n$ & Number of UEs probed \\
\hline$\tau$ & D2D probing time \\
\hline$\varepsilon \varepsilon_{(T x, R x)}$ & Energy efficiency of D2D communication between $T x$ and $R x$ \\
\hline$\varepsilon \varepsilon_{\operatorname{Init}(T x, R x)}$ & Initial $\varepsilon \varepsilon$ of D2D communication between $T x$ and $R x$ \\
\hline$\rho_{(T x, R x)}$ & Power consumption co-efficient between $T x$ and $R x$ \\
\hline$P_{(c k t)}$ & Power dissipated in the circuit \\
\hline$N$ & Upper limit for $\Phi, \Psi$, and $t_{(i(T x, R x))}$ \\
\hline $\mathbb{N}$ & Set of natural numbers \\
\hline
\end{tabular}

\subsubsection{SPL Parameters}

In this subsection, we define three SPL parameters, i.e., Social Friendship Index (SFI), Social Closeness Index (SCI), and Interest Similarity Index (ISI).

Social Friendship Index

The SFI describes the strength of the social relationships between the users based on their interaction history on various social networks. The higher the social network interaction, the closer friendship and consequently a higher SFI value. We categorized SFI into four classes: close friend, 
colleague, acquaintance, and stranger as given in Table 2. In the real system, the SCBS will assign SFI values based on the interaction history of users on various social network applications. However, for our simulations, we assume random values for SFI between the users as discussed in Section 5 .

Table 2. SFI values for different friendship classes.

\begin{tabular}{cc}
\hline Friendship Class & SFI Value \\
\hline Close friend & $1 \geq S F I \geq 0.75$ \\
Colleague & $0.75 \geq S F I \geq 0.50$ \\
Acquaintance & $0.50 \geq S F I \geq 0.25$ \\
Stranger & $S F I \leq 0.25$ \\
\hline
\end{tabular}

\section{Social Closeness Index}

The SCI characterizes the intimacy between the users in terms of the number of common friends in their friend-lists. A larger number of mutual friends between two users implies a stronger SCI between them. As the $S C I$ is based on the similarity between the friends' lists of the two users, which are finite sets, we utilize the well-known Jaccard coefficient method, which compares the elements of two sets (i.e., friends lists), and finds mutual and distinct friends. We define $S C I$ between $T x$ and $R x$ as a ratio of their common friends' to the total number of friends in their friends lists, calculated as given below [15]

$$
\operatorname{SCI}_{(T x, R x)}=\frac{\left(\Phi_{T x} \bigcap \Phi_{R x}\right)}{\left(\Phi_{T x} \cup \Phi_{R x}\right)}
$$

where $\Phi_{T x}$ and $\Phi_{R x}$ are the friends lists of $T x$ and $R x$, respectively.

Interest Similarity Index

The ISI determines the degree of interest of the users for similar contents. Users interested in the same contents are more likely to cooperate with each other in D2D communications even if they are not friends of each other. As we consider the interest lists of users as sequences of $1 \mathrm{~s}$ and $0 \mathrm{~s}$, the famous method to determine the similarity between such sequences is the weighted cosine similarity. Hence, we use it to determine the ISI between $T x$ and $R x$, which is defined as the ratio of their common interest to the root of the product of their individual interests [16] and is given by

$$
\operatorname{ISI}_{(T x, R x)}=\frac{\Psi_{T x R x}}{\sqrt{\Psi_{T x} \cdot \Psi_{R x}}}
$$

where $\Psi_{T x R x}$ represents the mutual interest list while $\Psi_{T x}$ and $\Psi_{R x}$ represent individual interest lists of $T x$ and $R x$, respectively. In case of no friend users in proximity (i.e., SFI $<0.25$ ), ISI can be used to select a D2D peer as a user could agree to share contents of common interest based on mutual reciprocity.

\subsubsection{PPL Parameters}

In this subsection, we define three PPL parameters, i.e., Encounter Duration, Distance between DUEs, and Number of DUEs.

\section{Encounter Duration}

Encounter Duration between $T x$ and $R x$ is the time when both are located in the coverage area of the same SCBS and encounter each other by getting close enough to be in D2D proximity of each other (i.e, $\left.D_{T x R x}<d_{t h}\right)$. Encounter duration between $T x$ and $R x$ is denoted by $r_{(T x, R x)}$ and is given by [17]

$$
(T x, R x)=\frac{\sum_{i=1}^{e} t_{i(T x, R x)}}{\Gamma}
$$


where $e$ is the number of encounters, $t_{i(T x, R x)}$ is the $i_{\text {th }}$ encounter time between $T x$ and $R x$, and $\Gamma$ is the total sample time, which is the total time during which $r_{(T x, R x)}$ is measured. The method to compute $r_{(T x, R x)}$ is illustrated by an example in Figure 2, where $r_{(T x, R x)}=\left(t_{1}+t_{2}+t_{3}\right) / \Gamma$. The rectangular boxes represent the activity time of $T x$ and $R x$, and $t_{1}, t_{2}$, and $t_{3}$ represent the time during which both $T x$ and $R x$ are active simultaneously in each other's proximity and, hence, encounter each other.

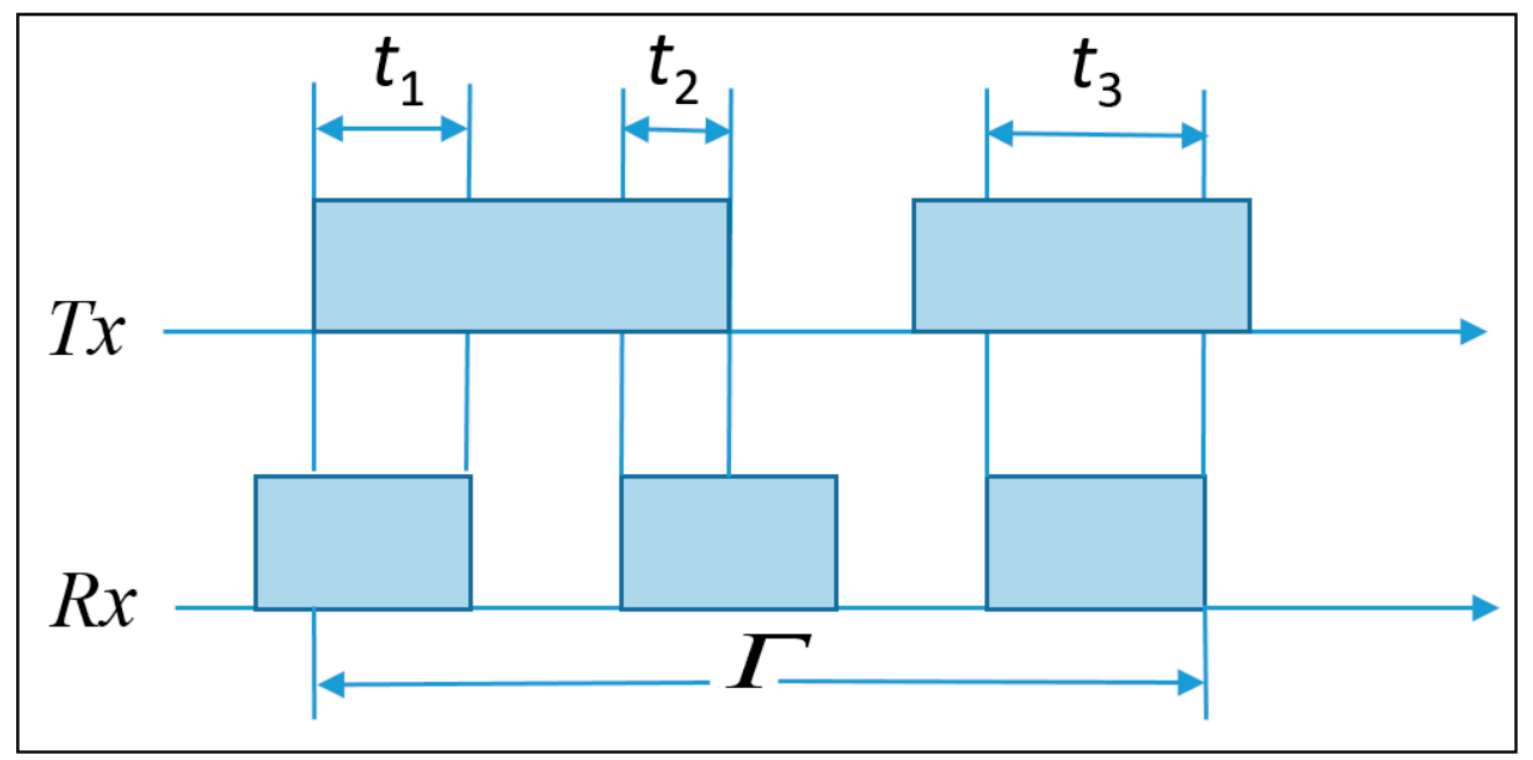

Figure 2. Encounter duration of $T x$ and $R x$.

\section{Distance between DUEs}

The distance $(D)$ between DUEs is the most important physical attribute. If we assume that $T x$ is currently located at polar coordinates $\left(X_{T x}, \theta_{T x}\right)$ while $R x$ is at $\left(X_{R x}, \theta_{R x}\right)$, where $0 \leq \theta_{T x}, \theta_{R x} \leq 2 \Pi$; then, the distance $D_{(T x, R x)}$ between $T x$ and $R x$ can be determined from their current locations [18] using

$$
D_{(T x, R x)}=\sqrt{X_{T x}^{2}+X_{R x}^{2}-2 X_{T x} X_{R x} \cos \left(\theta_{T x}-\theta_{R x}\right)},
$$

Number of DUEs

The number of DUEs $(m)$ also has a great impact on D2D communications. With a lower value of $m$, the number of friend DUEs are also fewer. Moreover, the DUEs are located far away from each other. Conversely, with the increase in $m$, the friend DUEs are increased, and the distance among the DUEs is decreased. Hence, we consider $m$ along with $r$ and $D$ in the $\mathrm{M}_{D P}$.

\section{Formulation of Social-Aware D2D Peer Selection Algorithm and Performance Indicators}

\subsection{Social-Aware D2D Peer Selection Algorithm}

The D2D peer selection is based on the social relationship between human users and the physical parameters of DUEs. The users who are friends on social networks and are of the same age group and profession are more likely to have similar choices. Moreover, for D2D communications, users also need to be located in close proximity. Therefore, we consider both social and physical attributes to perform D2D peer selection. For this purpose, we use the modified TOPSIS method to formulate the D2D peer selection problem.

TOPSIS is a popular MADM algorithm used to solve problems that involve the ranking of a set of alternatives based on multiple attributes of different natures. It efficiently integrates those attributes to decide the best among the available alternative solutions based on their comparison to the best (ideal 
positive) and worst (ideal negative) solutions. As social-aware peer selection is a ranking problem that combines the social and physical attributes of users to select the best one as the D2D peer. It does not involve maximization/minimization of an objective function to get an optimal solution. Therefore, the TOPSIS algorithm rather than the regular optimization algorithms could efficiently solve this problem. The proposed method works in the following way.

A generic decision matrix $\mathbf{M}_{D}$ is formed with UEs as alternatives in the rows and attributes as constraints in the columns, as presented in (5)

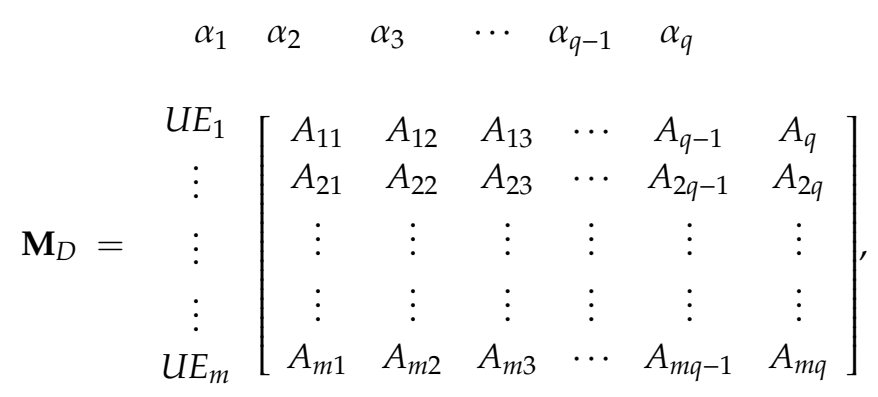

where $A_{i, j}$ is the value of attribute $\alpha_{j}$ for $U E_{i}$.

The proposed scheme exploits two types of parameters (i.e., social and physical); therefore, we construct two separate decision matrices, $\mathbf{M}_{D S}$ and $\mathbf{M}_{D P}$, based on the social and physical attributes, respectively. As the modified TOPSIS algorithm is uniformly applicable to both types of attributes, we present a generic mathematical model as presented in Equation (5) to (14) to describe the basic mechanism of the proposed scheme.

At this point, we exclude from $\mathbf{M}_{D S}$ the UEs with no social friendships (i.e., $S F I<0.25$ ) with the requesting DUE as they could not be suitable D2D peers. Similarly, we eliminate from $\mathbf{M}_{D P}$ the UEs that are located at a distance greater than the threshold $d_{t h}$ (i.e., $D>d_{t h}$ ) from the requesting UE. This significantly simplifies the task of peer selection. If there are no friendly UEs in the proximity, then the UEs are ranked based on the ISI defined in (2).

Now, the values for each attribute have different scales. Therefore, we normalize the decision matrix to a uniform scale. Moreover, we distinguish the attributes as desired and undesired based on their impact on the overall performance. The desired attributes are normalized as

$$
\left\|A_{i, j}^{d e s}\right\|=\frac{A_{i, j}}{\sum_{i=1}^{m} A_{i, j}} \text { for } i=1, \ldots, m \text { and } j=1, \ldots, q,
$$

while the undesired attributes are normalized as

$$
\left\|A_{i, j}^{\text {undes }}\right\|=1-\frac{A_{i, j}}{\sum_{i=1}^{m} A_{i, j}} \text { for } i=1, \ldots, m \text { and } j=1, \ldots, q,
$$

After normalization, the desired and undesired attributes have uniform scales. Therefore, we derive a general expression as given in (8) to simplify the forthcoming steps.

$$
\left\|A_{(i, j)}\right\|=\left\{\begin{aligned}
\left\|A_{i, j}^{\text {des }}\right\| & \text { for desired attributes } \\
\left\|A_{i, j}^{\text {undes }}\right\| & \text { for undesired attributes }
\end{aligned}\right.
$$

Based on the impact on performance, we assign weights, $W$, to each normalized attribute as

$$
A_{w(i, j)}=W_{i *}\left\|A_{(i, j)}\right\|, \text { where } \sum_{i=1}^{q} W_{i}=1,
$$

We assign weights to various attributes, as described in Section 5. 
Subsequently, we determine the ideal positive $\left(I_{j}^{+}\right)$solution, which is the maximum value of an attribute for a UE in the $\mathbf{M}_{D}$ as given by

$$
I_{j}^{+}=\max \left(A_{W(1, j)}, A_{W(2, j)}, \ldots \ldots A_{W(m, j)}\right) \text { for } j=1, \ldots, q,
$$

Similarly, the ideal negative solution, $I_{j}^{-}$, which is the minimum value of an attribute for a UE in $\mathbf{M}_{D}$, is computed as

$$
I_{j}^{-}=\min \left(A_{W(1, j)}, A_{W(2, j)}, \ldots \ldots A_{W(m, j)}\right) \text { for } j=1, \ldots, q,
$$

Each attribute is then compared with the ideal positive and negative solutions by calculating its distance from $I_{j}^{+}$and $I_{j}^{-}$(i.e., $D I_{j}^{+}$and $D I_{j}^{-}$) as given by

$$
\begin{aligned}
& D I_{j}^{+}=\sqrt{\sum_{j=1}^{q}\left(A_{w(i, j)}-I_{j}^{+}\right)^{2}}, \\
& D I_{j}^{-}=\sqrt{\sum_{j=1}^{q}\left(A_{w(i, j)}-I_{j}^{-}\right)^{2}},
\end{aligned}
$$

The alternative that has a minimum distance from $I_{j}^{+}$and maximum distance from $I_{j}^{-}$is considered as the most suitable solution. The mechanism of calculating the distance of each alternative solution from the positive and negative solutions is illustrated in Figure 3.

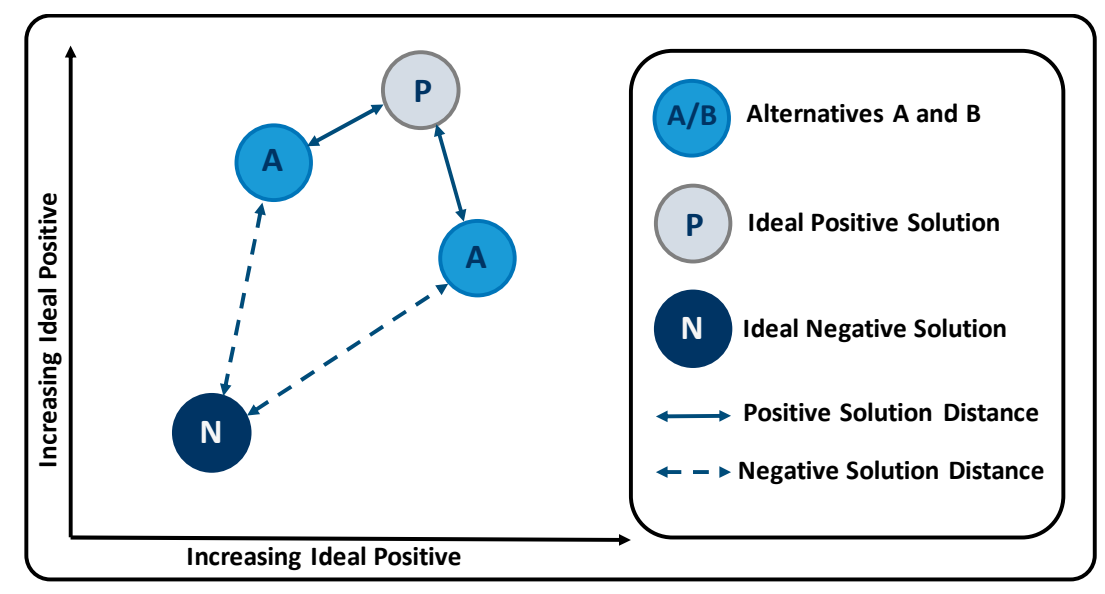

Figure 3. Distance of the alternative solutions from the positive and negative ideal solution.

Subsequently, we use $D I_{j}^{+}$and $D I_{j}^{-}$from (12) and (13) to compute the closeness coefficient of the $\mathrm{UE}_{i}$ (i.e., $\delta_{i}$ ) based on the distances from the ideal solutions as given by

$$
\delta_{i}=\frac{D I_{j}^{-}}{D I_{j}^{-}+D I_{j}^{+}} .
$$

Equation (14) is uniformly applicable to both social and physical attributes. Therefore, $\delta_{i}$ from $\mathbf{M}_{D S}$ is represented by $\delta_{s}$ and by $\delta_{p}$ from the $\mathbf{M}_{D P}$. We add $\delta_{s}$ and $\delta_{p}$ to get the cumulative closeness coefficient which is denoted by $\Delta$. Based on the values of $\Delta$, we sort the UEs in descending order. The UE with the highest $\Delta$ value is the best choice for D2D communications. 


\subsection{Performance Indicators}

In this subsection, we first compute $\gamma$ and $R$ for D2D communications. Based on that, we determine $\mathfrak{J}$ and $\varepsilon \varepsilon$ as given below.

\subsubsection{Signal to Noise Ratio}

We incorporate $\Delta$ to compute $\gamma$ at $R x$ (i.e., $\gamma(R x)$, which is related to the transmission power, path-loss coefficient, additive white Gaussian noise (AWGN) with zero mean, and the variance $\sigma$ [19] and is given by

$$
\gamma_{(R x)}=\frac{P_{(T x,)} h_{(T x, R x)} \Delta_{(T x, R x)}}{\sigma^{2} D_{(T x, R x)}^{\beta}},
$$

where $P_{(T x)}$ is the transmission power of $T x, h_{(T x, R x)}$ is the Rayleigh coefficient, $\Delta_{(T x, R x)}$ is the cumulative closeness coefficient between $T x$ and $R X$, and $\beta$ is the coefficient of path-loss.

\subsubsection{Data Rate}

We then use the computed $\gamma_{(R x)}$ from (15) to calculate $R$ between $T x$ and $R x$ (i.e., $R_{(T x, R x)}$ ) as given below

$$
R_{(T x, R x)}=B W \min \left\{\log _{2}\left(1+\gamma_{(R x)}\right),\right.
$$

where $B W$ is the available bandwidth.

Then, we compute $\mathfrak{I}$ and $\varepsilon \varepsilon$ for D2D communications in macro- and small-cells in (17) and (18) to evaluate the performance of our proposed scheme.

\subsubsection{Throughput}

Using $R_{(T x, R x)}$ from (16), we compute $\mathfrak{I}$ for the D2D communications between $T x$ and $R x$ (i.e., $\left.\mathfrak{J}_{(T x, R x)}\right)$, as given by [20]

$$
\mathfrak{J}_{(T x, R x)}=\frac{R_{(T x, R x)}\left(T_{T x}-n \tau\right)}{T_{T x}},
$$

where $T_{T x}$ is the transmission time of $T x$ to $R x, n$ is the number of DUEs probed during the peer-selection process, and $\tau$ denotes the time required to probe a $\mathrm{D} 2 \mathrm{D}$ peer.

\subsubsection{Energy Efficiency}

We calculate the $\varepsilon \varepsilon$ of D2D communications between $T x$ and $R x$ (i.e., $\varepsilon \varepsilon_{(T x, R x)}$ ) in small- and macro-cells using the following equation [21]

$$
\varepsilon \varepsilon_{(T x, R x)}=\frac{R_{(T x, R x)}}{P_{(T x)}+P_{c k t}}-\rho_{(T x, R x)} \varepsilon \varepsilon_{\text {Init }}
$$

where $P_{c k t}$ is the circuit power consumption, $\varepsilon \varepsilon_{\text {Init }}$ represents the initial energy efficiency of potential D2D peers which has a constant value given in Table 3, and $\rho_{(T x, R x)}$ is the power consumption co-efficient defined in (19). As in social-aware D2D communications, the transmit power depends on the social relationship strength between the two DUEs, the power consumption coefficient between $T x$ and $R x$ is modeled as an exponentially decaying function as given by [21]

$$
\rho_{(T x, R x)}=\exp \left(-\delta_{s(T x, R x)} S C I_{(T x, R x)}\right)
$$

where $\delta_{s(T x, R x)}$ is the social closeness coefficient between $T x$ and $R x$, which is generically defined in (14) for both the social and physical parameters. 


\section{Simulations and Numerical Results}

We performed simulations of the proposed scheme in MATLAB. We considered hexagonal small-cell scenarios with uniform UEs randomly distributed in the coverage areas of SCBS as shown in Figure 4. Moreover, we changed the coverage area and transmission power to simulate the MCBS scenario. The physical channel between $T x$ and $R x$ was modeled as $H_{(T x, R x)}=D_{(T x, R x)}^{\beta}\left|h_{T x, R x}\right|$, where $D_{(T x, R X)}$ is defined in (4), $h_{(T x, R X)}$ is the Rayleigh coefficient, and $\beta$ is the path-loss exponent. We assumed each UE has $Q$ number of social attributes such as $\alpha_{1}{ }^{\mathrm{s}}, \alpha_{2}{ }^{\mathrm{s}}, \ldots, \alpha_{Q}{ }^{\mathrm{s}}$ which include SFI, SCI, and ISI and physical attributes such as $\alpha_{1} \mathrm{p}, \alpha_{2} \mathrm{p}, \ldots, \alpha_{\mathrm{Q}}{ }^{\mathrm{p}}$ which include $r, D$, and $m$ as defined in Sections 3.2.1 and 3.2.2. We classified SFI, SCI, ISI, $r$, and $m$ as desired attributes and normalized them using (6). Similarly, $D$ was classified as an undesired attribute, and we normalized it using (7). Among the social attributes, the most important is SFI. Hence, we assigned it 0.5 weight, and 0.25 (i.e., $0.5 / 2$ ) to each of the remaining two social attributes. Our proposed algorithm worked even if there is no friend UE in the proximity by selecting a D2D peer based on common interests. Hence, it selected the UE with maximum ISI value. Similarly, for physical attributes, we assign 0.5 to $D$ as it is the most important physical attribute and 0.25 to each of the remaining two physical attributes.

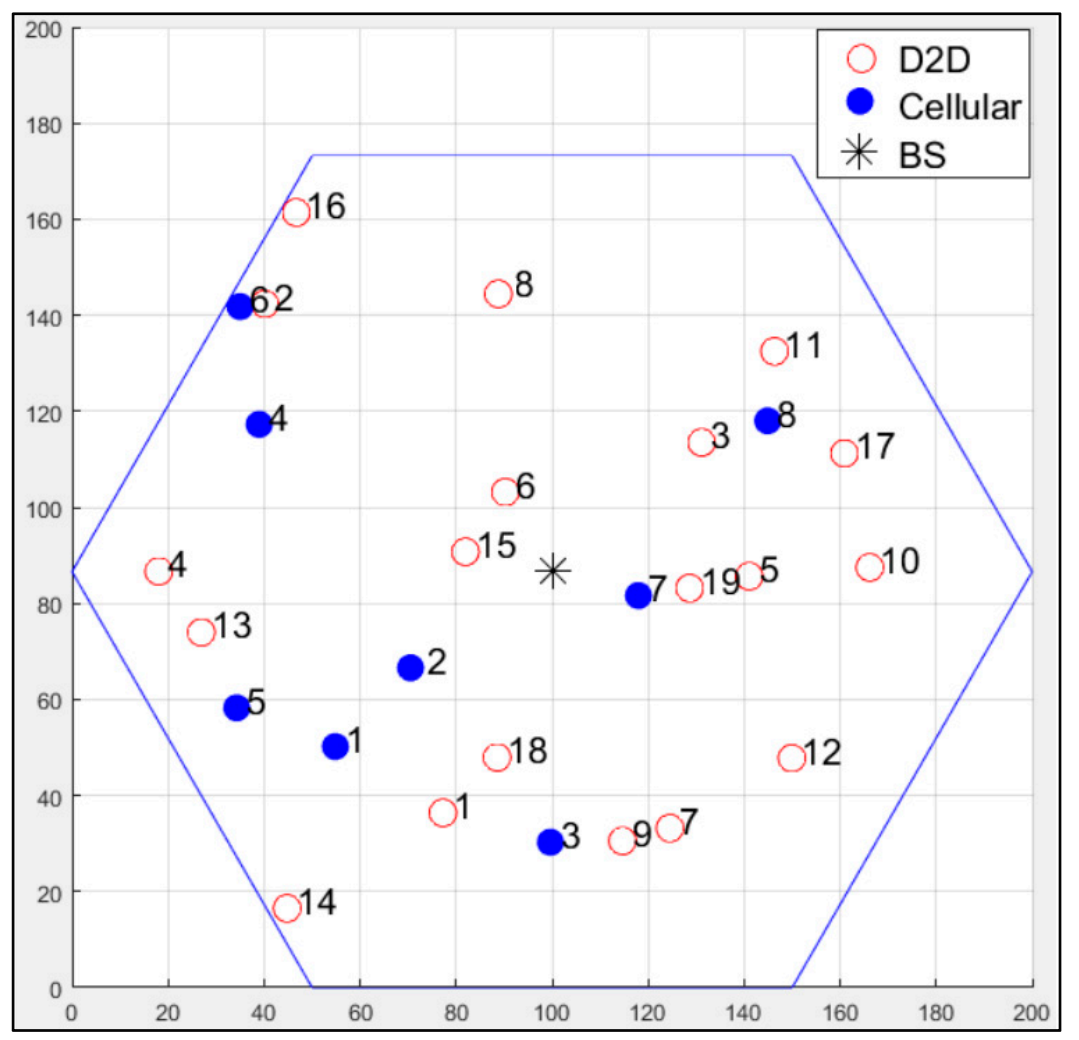

Figure 4. Proposed model with BS, DUEs, and CUEs.

For social attributes, we assigned $S F I$ value as a random number between 0 and 1 [18]. To calculate $S C I$, we assumed a friends' list (for $T x$ and $R x$, i.e., $\Phi_{T x}, \Phi_{R x}$ ) as $\Phi=\left\{F_{1}, F_{2}, \ldots, F_{N}\right\}$, where $F_{i} \in[1, \ldots, N]$ and $N \in \mathbb{N}$ [22]. This meant that $T x$ and $R x$ can have any number of friends in the range of 1 and $N$. For any value of $N$, the value of $S C I$ ranges between 0 and 0.5 . However, for simplicity, we assume $N$ in the range of 1 to 20. Similarly, to compute ISI, we assumed $\Psi$ (individual interests list for $T x$ and $R x$-i.e., $\Psi_{T x}, \Psi_{R x}$ - or mutual interests' list $\left.\Psi_{T x R x}\right)$ as $\Psi=\left\{I_{1}, I_{2}, \ldots, I_{N}\right\}$, where $I_{i} \in[0,1]$ and $N \in \mathbb{N}[22,23]$. For physical attributes, we calculated $r$ by assuming $\Gamma=20 \mathrm{~s}$, and $\sum_{i=1}^{e} t_{i(T x, R x)} \in[0, N]$ and $N \in \mathbb{N}$ [24]. Similarly, $D$ was calculated based on the polar coordinates of uniformly distributed UEs in the coverage area using (5) [17]. 
We evaluated the performance of the proposed scheme by showing the numerical results in terms of the algorithm computation time, selection of best peer, $\mathfrak{I}$, and $\varepsilon \varepsilon$ of D2D communications as given in the subsequent subsections. The parameters used for simulation are given in Table 3.

Table 3. Simulation Parameters.

\begin{tabular}{|c|c|}
\hline Parameter & Value \\
\hline Network & Small- and macro-cell \\
\hline$m$ & 5 to 50 \\
\hline Carrier frequency & $2 \mathrm{GHz}$ [25] \\
\hline Small-cell radius & $100 \mathrm{~m} \mathrm{[24]}$ \\
\hline Macro-cell radius & $1000 \mathrm{~m}$ \\
\hline$Q$ & 3 \\
\hline$d_{t h}$ & $50 \mathrm{~m} \mathrm{[26]}$ \\
\hline$\beta$ & $3[1]$ \\
\hline SFI & 0 to 1 [17] \\
\hline$N$ & 1 to 20 [24] \\
\hline$T_{T x}$ & 1 to $20 \mathrm{~s}$ [20] \\
\hline$\Gamma$ & $20 \mathrm{~s}$ [24] \\
\hline$\tau$ & $0.01 \mathrm{~s} \mathrm{[20]}$ \\
\hline$\varepsilon \varepsilon_{\text {Init }}$ & $1 \mathrm{Kbps} / \mathrm{W}[21]$ \\
\hline$P_{c k t}$ & $0.01 \mathrm{~W}[21]$ \\
\hline $\mathrm{UE} P_{(T x)}$ & $23 \mathrm{dBm}[26]$ \\
\hline SCBS $P_{(T x)}$ & $40 \mathrm{dBm}[25]$ \\
\hline $\operatorname{MCBS} P_{(T x)}$ & $46 \mathrm{dBm}[26]$ \\
\hline Fading & Rayleigh flat fading [13] \\
\hline Number of experiments & 50 \\
\hline User distribution & Uniform \\
\hline
\end{tabular}

\subsection{Computation Time}

Figure 5 shows the computation time for the proposed scheme with respect to $m$. The results are compared to the well-known Hungarian algorithm used for selection and assignment problems. The two algorithms are run on the same PC with 50 experiments for each value of $m$. The computation time of the proposed scheme is much lower and shows a marginal increase with the increase of $m$. However, in the case of the Hungarian scheme, it is much higher and is significantly increased when $m$ is increased. In the proposed scheme, we exclude the unfriendly and distant UEs at the initial time. This significantly decreases the computational time in contrast to the comparing scheme.

\subsection{Best Peer Selection}

Figures 6-8 illustrate the peer selection mechanism of the proposed scheme based on social, physical, and combined attributes, respectively. In this scenario, we maintained $m$ as a constant (i.e., five) and run the algorithm for 10 iterations. For the results in Figure 6, we took into account the social attributes of DUEs only. We applied the algorithm formulated in Section 4.1 to compute the closeness coefficient for social attributes (i.e., $\delta_{s}$ ). We ranked the DUEs based on the value $\delta_{s}$ and selected the best one as D2D peer. It was clear that, in each iteration, the UE with the maximum value of $\delta_{s}$ was selected as marked with a blue circle in the figure. 


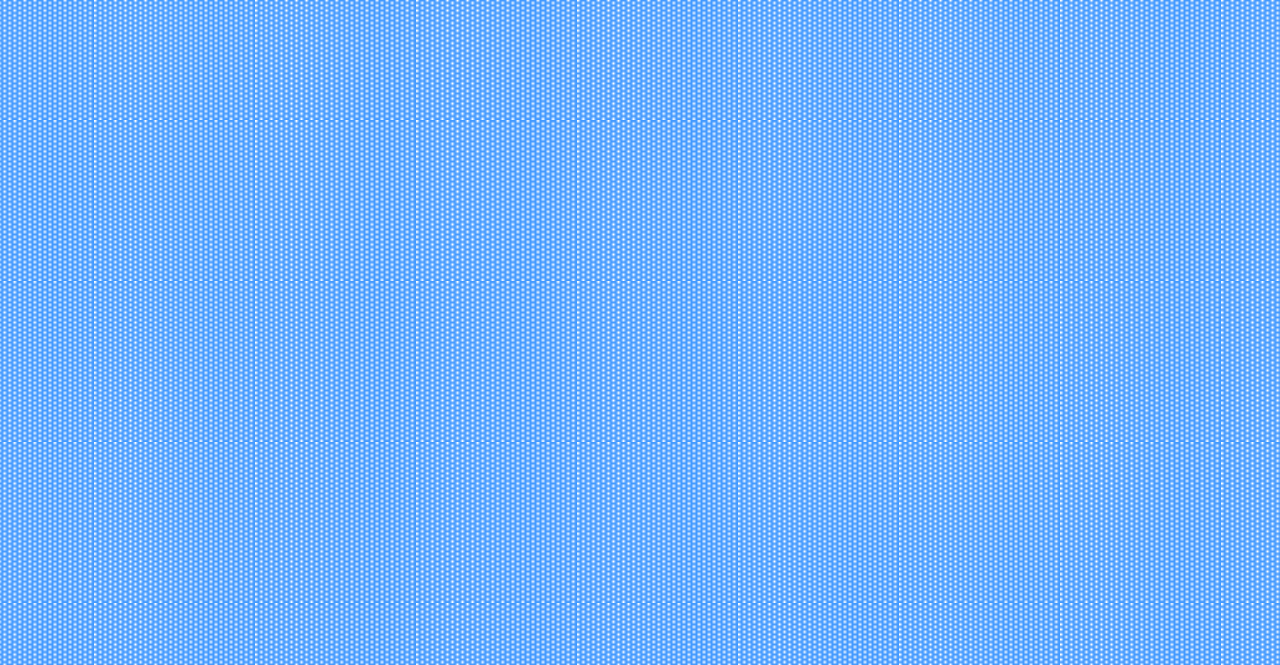

Figure 5. Computation time comparison of the proposed scheme with the Hungarian algorithm vs. $m$.

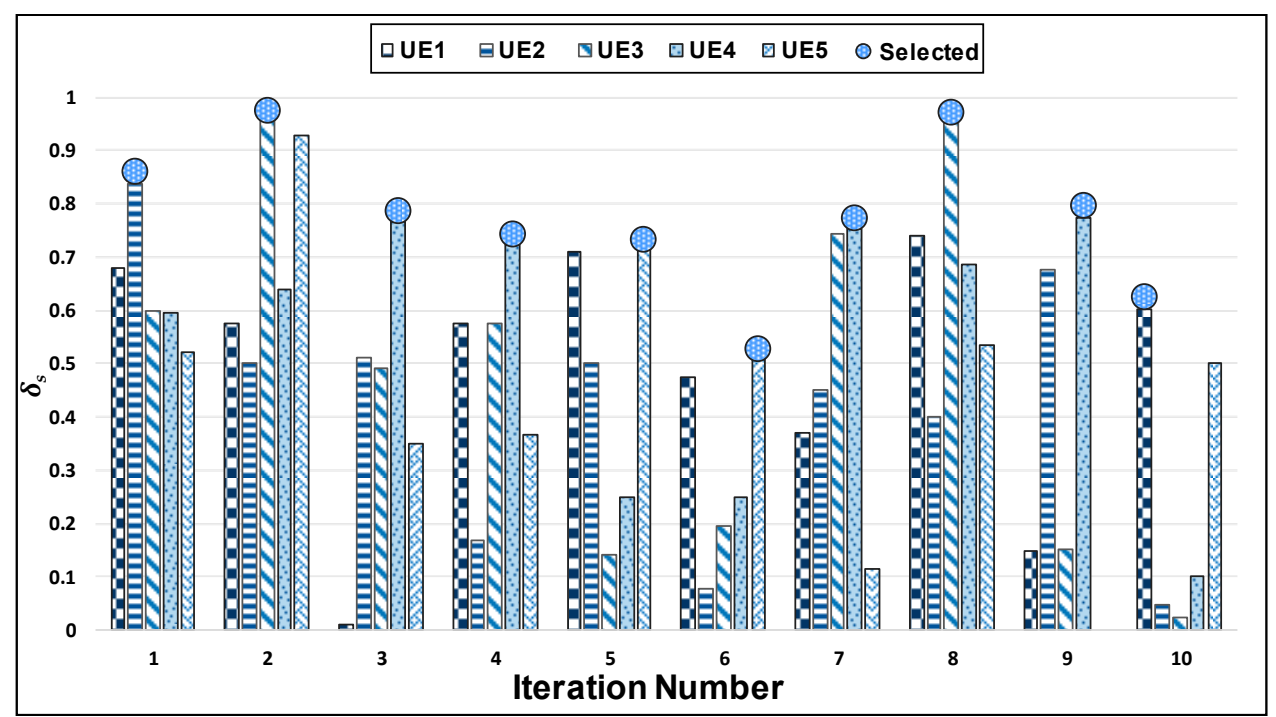

Figure 6. Selection of UE with the best $\delta_{s}$ values.

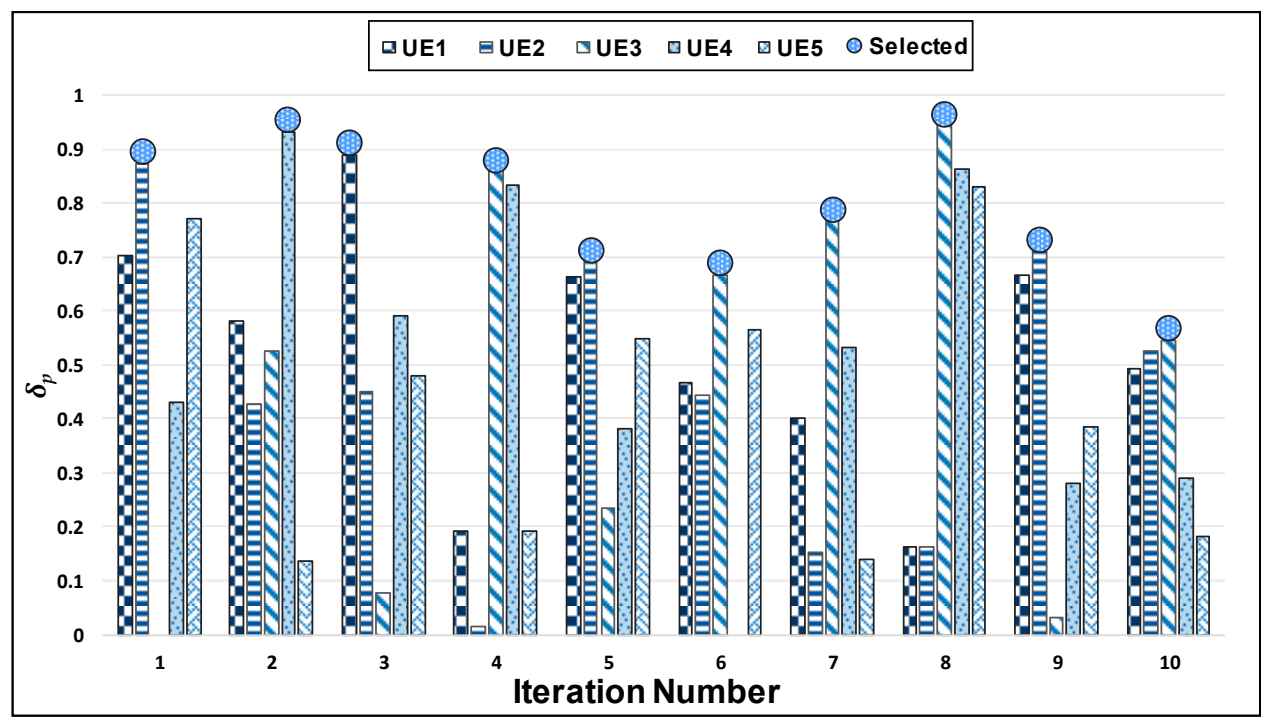

Figure 7. Selection of UE with the best $\delta_{p}$ values. 


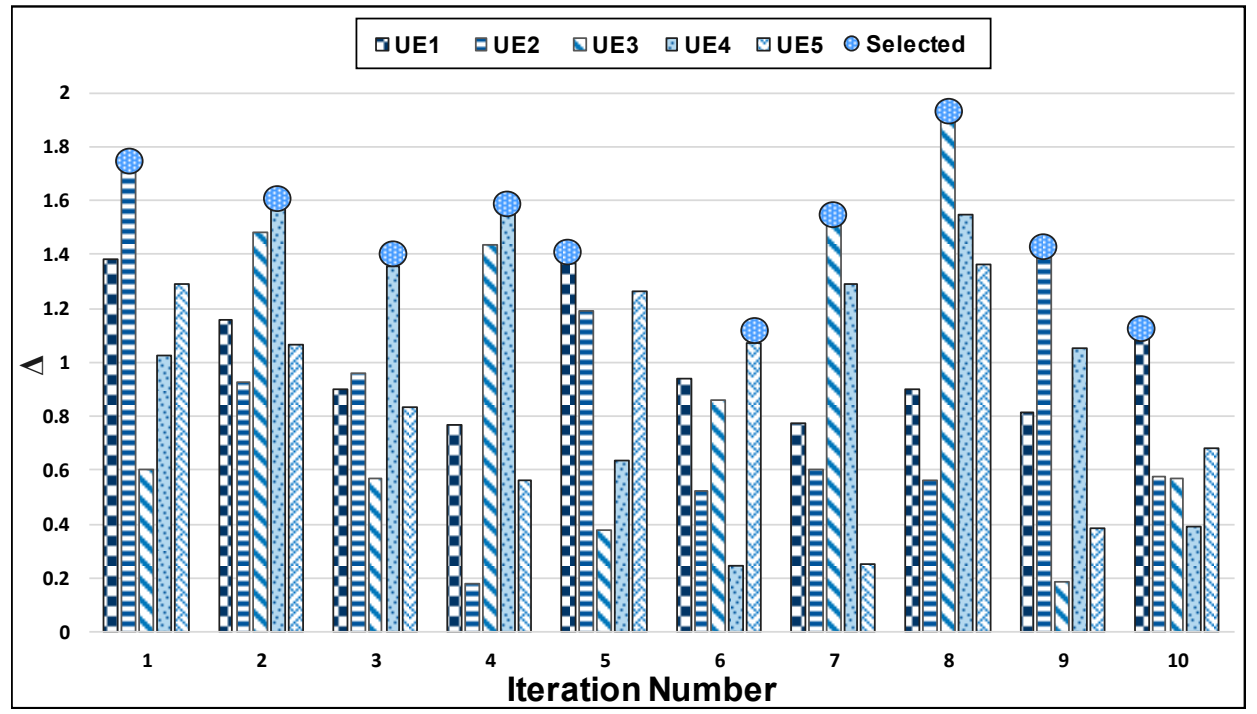

Figure 8. Selection of the UE with best $\Delta$ values.

Similarly, for the results in Figure 7, we only took into account the physical attributes of the DUEs. We followed the same procedure to compute the closeness coefficient for physical attributes (i.e., $\delta_{p}$ ). We ranked the DUEs based on the value $\delta_{p}$ and selected the best one as the D2D peer. It was clear that, in each iteration, the UE with the maximum value of $\delta_{p}$, was selected as marked with a blue circle in the figure.

Figure 8 shows the D2D peer selection based on the combined social and physical attributes. It can be observed that in each iteration, DUE with a maximum $\Delta$ was selected for the D2D communication. As $\Delta$ is obtained from the summation of $\delta_{s}$ and $\delta_{p}$, whose values lie between 0 and 1 , the value of $\Delta$ exceeded 1 in most of the cases. Moreover, the social and physical parameters had different impacts on the performance of D2D communications. Therefore, a DUE could have had the maximum value of $\delta_{p}$ but was not selected as the best D2D peer because the value of $\delta_{s}$ was smaller, and vice versa. This phenomenon was observed in the $5^{\text {th }}$ iteration, where UE5 was selected based on the highest value of $\delta_{s}$ and UE2 was selected based on the highest value of $\delta_{p}$ in Figures 6 and 7, respectively. However, the value of $\Delta$ was maximum for UE1. Therefore, it was selected as the final best D2D peer. The maximum value of $\Delta$ led to higher $\gamma, R$, and eventually the best $\mathfrak{~}$.

\subsection{Throughput}

Figure 9 illustrates the throughput derived in (17). The proposed scheme is compared with three schemes; i.e., Random, Social-, and Physical-TOPSIS. The Random scheme selects the D2D peer randomly without considering social or physical parameters. The Social-TOPSIS scheme only performs peer selection based on social parameters using unmodified TOPSIS algorithm. The Physical-TOPSIS uses the same algorithm for peer selection taking into account only the physical parameters. Hence, the two methods use $\delta_{s}$ and $\delta_{p}$ to compute $\mathfrak{I}$ instead of $\Delta$ in (17), respectively. Finally, the proposed scheme selects the best D2D peer based on both the social and physical attributes (i.e., $\Delta$ ) using the modified TOPSIS algorithm.

It is clear from the graph that the D2D throughput for the proposed scheme improves significantly, as it combines both the social and physical attributes of UEs and applies the modified TOPSIS algorithm to perform peer selection. Moreover, when the number of UEs is increased, the distance between UEs decreases and the number of friendly UEs increases. Both these parameters affect the $\gamma, R$, and ultimately the $\mathfrak{I}$ as obtained using (15) to (17). The Random scheme gives very low throughput, as it selects D2D peer randomly without any manipulation of social and physical attributes. The Socialand Physical-TOPSIS schemes are better than the random scheme as these two methods select the D2D peer based on one type of attribute. The Physical-TOPSIS is slightly better than its counterpart, as 
it considers the minimum value of $D$ for the computation of $\gamma$. However, both methods have lower throughput compared to the proposed scheme, which reveals that only these types of attributes are not sufficient for D2D peer selection.

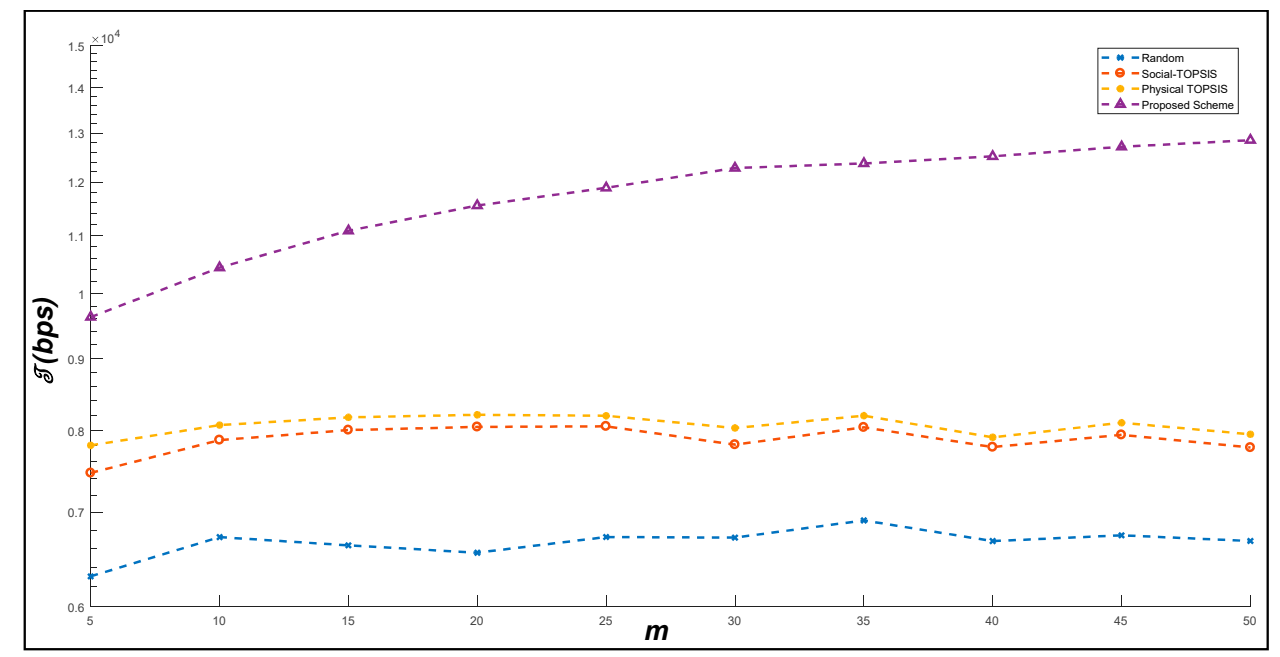

Figure 9. D2D throughput vs. $m$.

\subsection{Energy Efficiency}

Figure 10 demonstrates the performance of the proposed scheme in terms of $\varepsilon \varepsilon$ computed by (18). Simulation experiments are performed for the macro- and small-cell with $m$. The results show that the proposed scheme is energy efficient and suitable for small-cell D2D communication. The energy efficiency is increased with the increase of $m$ in both the scenarios, as the number of UEs with social friendship is also increased. Moreover, our proposed scheme focuses on D2D peer selection in small-cell scenarios; therefore, it is clear from the results that the small-cell scenario has much better $\varepsilon \varepsilon$ than the macro-cell scenario. The reason is that the UEs are much closer to each other in the small-cells than in macro-cells. Conversely, in the macro-cell, there are few UEs with social friendship in the proximity as the coverage area is wide. Therefore, higher transmit power is required for D2D communications, which reduces its $\varepsilon \varepsilon$.

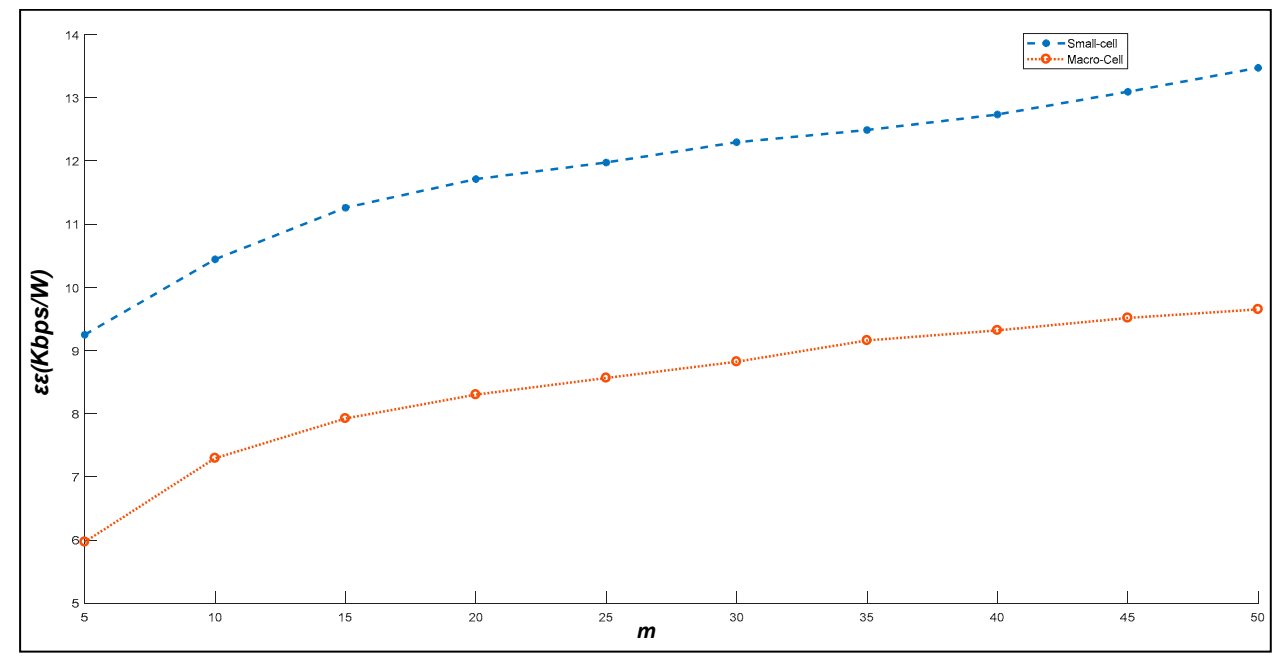

Figure 10. D2D energy efficiency of small-cell and macro-cell scenarios vs. $\mathrm{m}$. 


\section{Conclusions and Future Work}

In this paper, we proposed a peer selection algorithm for D2D communications in dense small-cell networks. We exploited both the social and physical parameters of the UEs to improve the willingness of the users and enhance the performance of the overall D2D communications in terms of throughput and energy efficiency. We exploited a modified TOPSIS algorithm to determine the most suitable D2D peer. The simulation results for the proposed scheme are plotted to demonstrate its efficiency in terms of the computation time, selection of UEs with the best values of $\delta_{s}, \delta_{p}$, and $\Delta, \mathfrak{I}$ and $\varepsilon \varepsilon$ of the D2D communications. The selection of UE with the best value of $\Delta$ ultimately achieved the best throughput for D2D communications between the requesting and selected DUEs. Similarly, the proposed small-cell paradigm was more energy-efficient as compared to the macro-cell system. In the future, we aim to consider realistic mobility traces and analyze the impact of various social and physical parameters on the D2D peer selection process. Similarly, we can further improve the performance by incorporating user preferences and dynamically assigning weights based on user application requirements to select a desired D2D peer. Moreover, the proposed work can be extended to scenarios where the DUEs were connected to different SCBSs.

Author Contributions: A.N. performed computer simulations and wrote the paper, H.-S.C. supervised the research and participated in problem formulation, data analysis and revision process.

Funding: This research was supported by the BK21 Plus project funded by the Ministry of Education, Korea (21A20131600011).

Conflicts of Interest: The authors declare no conflict of interest.

\section{References}

1. Zhang, Z.; Zeng, T.; Yu, X.; Sun, S. Social-aware D2D Pairing for Cooperative Video Transmission Using Matching Theory. Mob. Netw. Appl. 2018, 23, 639-649. [CrossRef]

2. Cisco. Cisco Visual Networking Index: Global Mobile Data Traffic Forecast Update, 2016-2021 White Paper. 28 March 2017. Available online: https://www.cisco.com/c/en/us/solutions/collateral/service-provider/visualnetworking-index-vni/mobile-white-paper-c11-520862.html (accessed on 15 October 2018).

3. Laya, A.; Wang, K.; Widaa, A.A.; Alonso-Zarate, J.; Markendahl, J.; Alonso, L. Device-to-device communications and small cells: Enabling spectrum reuse for dense networks. IEEE Wirel. Commun. 2014, 21, 105-2014. [CrossRef]

4. Diaz, C.G.; Saad, W.; Maham, B.; Niyato, D.; Madhukumar, A.S. Strategic device-to-device communications in backhaul-constrained wireless small cell networks. In Proceedings of the 2014 IEEE Wireless Communications and Networking Conference (WCNC), Istanbul, Turkey, 6-9 April 2014.

5. Tsolkas, D.; Passas, N.; Merakos, L. Device discovery in LTE networks: A radio access perspective. Comput. Netw. 2016, 106, 259-2016. [CrossRef]

6. Radwan, A.; Domingues, M.F.; Rodriguez, J. Mobile Caching-enabled Small-cells for Delay-tolerant e-Health Apps. In Proceedings of the IEEE International Conference on Communications Workshops (ICC Workshops), Paris, France, 21-25 May 2017.

7. Tzeng, G.-H.; Huang, J.-J. TOPSIS and Vikor. In Multiple Attribute Decision Making: Methods and Applications; CRC Press: New York, NY, USA, 2011; pp. 69-79.

8. Wang, L.; Stuber, G.L. Pairing for resource sharing in cellular device-to-device underlays. IEEE Netw. 2016, 30, 128-2016. [CrossRef]

9. Liu, C.-P.; He, C.-G.; Meng, W.-X.; Han, S. A design of D2D-pairing scheme on Voronoi diagram. In Proceedings of the 2017 13th International Wireless Communications and Mobile Computing Conference (IWCMC), Valencia, Spain, 26-30 June 2017.

10. Mao, H.; Feng, W.; Zhao, Y.; Ge, N. Joint Social-Position Relationship Based Cooperation among Mobile Terminals. IEEE Commun. Lett. 2014, 18, 2165-2168. [CrossRef]

11. Prasad, A.; Samdanis, K.; Kunz, A.; Song, J. Energy efficient device discovery for social cloud applications in 3GPP LTE-advanced networks. In Proceedings of the IEEE Symposium on Computers and Communications (ISCC), Funchal, Portugal, 23-26 June 2014. 
12. Gao, Y.; Xiao, Y.; Wu, M.; Xiao, M.; Shao, J. Dynamic Social-Aware Peer Selection for Cooperative Relay Management with D2D Communications. IEEE Trans. Commun. 2019. [CrossRef]

13. Yan, J.; Wu, D.; Zhang, C.; Wang, H.; Wang, R. Socially aware D2D cooperative communications for enhancing Internet of Things application. EURASIP J. Wirel. Commun. Netw. 2018, 2018, 132. [CrossRef]

14. Chen, X.; Proulx, B.; Gong, X.; Zhang, J. Exploiting Social Ties for Cooperative D2D Communications: A Mobile Social Networking Case. IEEE/ACM Trans. Netw. 2015, 23, 1484-2015. [CrossRef]

15. Niwattanakul, S.; Singthongchai, J.; Naenudorn, E.; Wanapu, S. Using of Jaccard Coefficient for Keywords Similarity. In Proceedings of the International MultiConference of Engineers and Computer Scientists, Hong Kong, China, 13-15 March 2013.

16. Li, Y.; Zhang, Z.; Wang, H. Transmission Mode Selection and Interference Mitigation for Social Aware D2D Communication. In Proceedings of the IEEE Global Communications Conference (GLOBECOM), Washington, DC, USA, 4-8 December 2016.

17. Zhu, K.; Zhi, W.; Zhang, L.; Chen, X.; Fu, X. Social-Aware Incentivized Caching for D2D Communications. IEEE Access 2016, 4, 7585-7593. [CrossRef]

18. Chiti, F.; Fantacci, R.; Pierucci, L. Social-Aware Relay Selection for Cooperative Multicast Device-to-Device Communications. Future Internet 2017, 9, 92. [CrossRef]

19. Zhang, M.; Chen, X.; Zhang, J. Social-aware Relay Selection for Cooperative Networking: An Optimal Stopping Approach. In Proceedings of the 2014 IEEE International Conference on Communications Workshops (ICC), Sydney, Australia, 10-14 June 2014.

20. Chen, X.; Gong, X.; Yang, L.; Zhang, J. A Social Group Utility Maximization Framework with Applications in Database Assisted Spectrum Access. In Proceedings of the 33rd Annual IEEE International Conference on Computer Communications (IEEE INFOCOM), Toronto, ON, Canada, 27 April-2 May 2014.

21. Yang, L.; Wu, D.; Xu, S.; Cai, Y. Social-aware energy-efficient joint mode selection and link allocation in D2D communications. In Proceedings of the International Conference on Wireless Communications \& Signal Processing (WCSP), Yangzhou, China, 13-15 October 2016.

22. Wang, R.; Wu, J.; Yan, J. Resource Allocation for D2D-Enabled Communications in Vehicle Platooning. IEEE Access 2018, 6, 50537-52018. [CrossRef]

23. Wang, Y.; Chen, X.; Jin, Q.; Ma, J. LIP3: A Lightweighted Fine-Grained Privacy-Preserving Profile Matching Mechanism for Mobile Social Networks in Proximity. In Proceedings of the International Conference on Algorithms and Architectures for Parallel Processing, Zhangjiajie, China, 18-20 November 2015.

24. Bui, M.-P.; Vo, N.-S.; Nguyen, T.-T.; Tran, Q.-N.; Tran, A.-T. Social-Aware Caching and Resource Sharing Optimization for Video Delivering in 5G Networks. In Proceedings of the Qshine 2018: Quality, Reliability, Security and Robustness in Heterogeneous Systems, Ho Chi Minh City, Vietnam, 3-4 December 2018.

25. Wang, Z.; Shen, C. Small Cell Transmit Power Assignment Based on Correlated Bandit Learning. IEEE J. Sel. Areas Commun. 2017, 35, 1045-2017. [CrossRef]

26. Wu, D.; Wu, J.; Wang, H.; Wang, R. User Satisfaction-Aware Resource Allocation for D2D Enhanced Communication. IEEE Access 2018, 7, 539-2018. [CrossRef]

(C) 2019 by the authors. Licensee MDPI, Basel, Switzerland. This article is an open access article distributed under the terms and conditions of the Creative Commons Attribution (CC BY) license (http://creativecommons.org/licenses/by/4.0/). 